\title{
Laboreal
}

Volume $10 \mathrm{~N}^{\circ} 1$ | 2014

Análise ergonómica do trabalho e formação

\section{La "Didáctica psicológica. Aplicación a la didáctica de la psicología de Jean Piaget" de Aebli, un enfoque y un autor olvidados}

A "Didática psicológica. Aplicação da psicologia de Jean Piaget à didática", por Aebli ; uma abordagem e um autor esquecidos

La "Didactique psychologique. Application à la didactique de la psychologie de Jean Piaget" de Aebli, une approche et un auteur oubliés

The "Psychological didactics. Application of the Jean Piaget psychology to the didactics", by Aebli; the forgotten approach of a forgotten author

\section{Janine Rogalski}

\section{OpenEdition}

\section{Journals}

Edición electrónica

URL: http://journals.openedition.org/laboreal/5452

DOI: 10.4000/laboreal.5452

ISSN: 1646-5237

Editor

Universidade do Porto

Referencia electrónica

Janine Rogalski, «La "Didáctica psicológica. Aplicación a la didáctica de la psicología de Jean Piaget" de Aebli, un enfoque y un autor olvidados », Laboreal [En línea], Volume $10 N^{0} 1$ | 2014, Publicado el 01 julio 2014, consultado el 23 septiembre 2020. URL : http://journals.openedition.org/laboreal/5452 : DOI : https://doi.org/10.4000/laboreal.5452

Este documento fue generado automáticamente el 23 septiembre 2020.

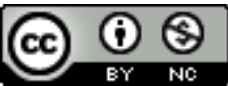

Laboreal está licenciado com uma Licença Creative Commons - Atribuição-NãoComercial 4.0 Internacional. 


\title{
La "Didáctica psicológica. Aplicación a la didáctica de la psicología de Jean Piaget" de Aebli, un enfoque y un autor olvidados
}

\author{
A "Didática psicológica. Aplicação da psicologia de Jean Piaget à didática", por \\ Aebli; uma abordagem e um autor esquecidos \\ La "Didactique psychologique. Application à la didactique de la psychologie de \\ Jean Piaget" de Aebli, une approche et un auteur oubliés \\ The "Psychological didactics. Application of the Jean Piaget psychology to the \\ didactics", by Aebli; the forgotten approach of a forgotten author
}

Janine Rogalski

\section{REFERENCIA}

Artigo original : Aebli, H. (1951). Didactique psychologique. Application à la didactique de la psychologie de Jean Piaget. Neuchâtel : Delachaux \& Niestlé.

\section{NOTA DEL EDITOR}

http://dx.doi.org/10.15667/laborealx0114jres

Las didácticas disciplinarias y profesionales contribuyen junto a la ergonomía, la sociología y la psicología del trabajo en el desarrollo de los conocimientos del análisis de la actividad en situación real en la escuela y en el trabajo. Los investigadores de dicho campo precisan raramente el origen de sus fuentes, tal vez porque su campo de investigación es reciente. En este artículo recordaremos lo que se le debe a un 
investigador cuyos trabajos precursores son poco conocidos o ignorados: Hans Aebli (1923-1990).

Hans Aebli publica en 1951 la obra "Didáctica psicológica. Aplicación a la didáctica de psicología de Jean Piaget", el mismo año en el que defiende su tesis con Piaget. Se trata de su primer libro y el único que existe en francés [1]. La característica original de esta publicación es no solo la de deducir una didáctica de la teoría psicológica de Piaget, sino también de demostrar experimentalmente la eficacia de este enfoque en un objeto de enseñanza matemática.

Sin embargo, como lo señalan Régis Ouvrier-Bonnaz y Marianne Lacomblez (2014), "cuando los pedagogos se interesaron en Piaget, fue sobre todo la dimensión epistemológica de sus investigaciones le que les llamó su atención, sin retomar ni transmitir los trabajos de Aebli". El término de "transmitir" aparece aquí importante pues esta contribución se hará en referencia a los trabajos franceses en pedagogía o en didáctica de las matemáticas, pero esencialmente en lo que corresponde a su existencia y no a su contenido didáctico salvo excepción [2]. La cuestión es porque esta propuesta de apoyar cada didáctica por el aporte teórico piagetiano no actuó de manera directa. Es una cuestión específicamente en didáctica de las matemáticas : al menos un debate crítico hubiese podido ser iniciado a partir de allí. Para tratar esta cuestión, situaremos brevemente "Didáctica psicológica" en la vida profesional de Aebli. Abordaremos por un lado lo que es para él la "didáctica" y cómo la sitúa en relación con las ciencias de la educación, y por otro lado su significación posterior en didáctica de las matemáticas. Después de su crítica de las suposiciones psicológicas de la "didáctica tradicional" y de la de la "escuela activa", presentaremos el proceso de elaboración por Aebli de un método didáctico a partir del marco teórico de Piaget y las líneas del experimento que presenta para ilustrar sus propuestas. Concluiremos intentando explicar por qué el balance que pretendió elaborar Aebli no jugó el rol precursor que podría haber desempeñado.

\section{La didáctica psicológica durante los estudios universitarios de Hans Aebli}

El contenido de su primera obra está marcado por experimentos realizados en el contexto piagetiano. Se apoya también en conocimientos de la propia experiencia de Aebli, maestro de primaria. Piaget insiste en su prefacio en la importancia de esa doble calificación de psicólogo y de educador, para deducir una utilización didáctica de los trabajos psicológicos. Además, en el curso de su periodo de estudios en Minneapolis a finales de los años 40, Aebli "se confrontó con el enfoque pragmático americano a través del filósofo de la educación John Dewey" (documento web de la Fundación Aebli Näf : http://www.ans.ch/fr/).

Los trabajos de Aebli que siguieron fueron marcados por varias características. Por un lado se alejará del marco constructivista piagetiano (Aebli, 1978) en su análisis del desarrollo del pensamiento del niño, introduciendo en particular la dimensión de interiorización del conocimiento conocidas por el maestro. Extenderá así sus trabajos hacia el análisis de la educación y de la formación de los educadores. Por otro lado publicará prácticamente en alemán (dadas su pertenencia institucional [3]) y desafortunadamente- ninguna de sus obras será traducida en francés. Su obra inicial se volverá así en Francia un "punto aislado" de la didáctica desarrollada por Aebli, 
mientras que en Alemania y en Suiza germanófona tendrá una posteridad bien establecida.

\section{La didáctica : ciencia y método de investigación de las disciplinas escolares}

Aebli define la didáctica como "una ciencia auxiliar de la pedagogía a la cual esta última delega, para la realización de detalle, las tareas educativas más generales" (introd. P. 1). Precisa los objetivos (problemas) : "toda didáctica debe definir [...] no solo cómo los alumnos "conocen cierta materia [primer problema didáctico], sino también cómo la aprenden [segundo problema didáctico] [...] Le incumbe a la didáctica además el hecho de estudiar las condiciones más favorables a los procesos de formación [tercer problema didáctico]".

Insiste en la dimensión metodológica de la didáctica y su relación con una psicología : “ todo método de educación es solidario de una psicología del niño y del pensamiento, a menudo no explicitada", mientras que la didáctica científica se da como objetivo deducir las medidas metodológicas más indicadas para provocar el conocimiento psicológico de los procesos de formación intelectual" (p. 2).

Como lo recuerda en el prólogo : "solo una psicología tal como la de Jean Piaget [...] aporta los conceptos necesarios a la solución de tales problemas didácticos".

Lo singular del término "didáctica" indica el carácter genérico del principio piagetiano en la esencia de la "Didáctica psicológica" : "pensar es operar". Sin embargo, Aebli precisa que el principio debe ser declinado según las diferentes disciplinas escolares y lo desarrolla en el caso de las matemáticas (de la escuela primaria).

\section{De la insuficiencia de la "didáctica tradicional" y del lugar de la "psicología tradicional" en los principios de la "escuela activa", a la psicología piagetiana fundadora de la "didáctica científica"}

La parte histórica de la obra de Aebli (pp. 5-37) pone en relieve en primer lugar que la "didáctica tradicional" se fundamenta en el "principio de la intuición". Esta concepción "sensualista-empirista" [4] se basa en la psicología tradicional empirista, incapaz de darse cuenta siquiera de la adquisición de imágenes mentales. La "didáctica de la escuela activa" se analiza a través de obras de teóricos "cuyos trabajos han dado lugar a realizaciones prácticas y que han planteado la psicología en la cual se basa su pedagogía". A la de W. A. Lay [5] le hace falta una concepción de la naturaleza activa del pensamiento, aunque considera precisamente al niño como "miembro de una comunidad de la cual padece las acciones y sobre la cual reacciona" (p. 21). Una parte importante se le dedica a Dewey con el fin de discutir de las didácticas basadas en una "interpretación instrumental del pensamiento" [6]. El pensamiento es ciertamente una herramienta, pero -critica Aeblimás allá del análisis de la función del pensamiento, hace falta la cuestión de la naturaleza intrínseca que la hace operatoria. 


\section{En el fundamento de la didáctica de Aebli, la psicología piagetiana}

El centro de la obra (pp. 39-196) es la explicitación de cómo la didáctica puede/debe alimentarse de la psicología piagetiana. En esta Parte psicológica (pp. 39-71 [7]), Aebli comienza con la interpretación de Piaget de la imagen mental como interiorización de la acción, que se opone a la concepción de la psicología tradicional ; examina luego la actividad intelectual del alumno en la escuela tradicional desde el punto de vista de la teoría de la interiorización (La imagen y la operación, pp. 40-48). Sigue el desarrollo de los conceptos de hábitos tal como se pueden adquirir en la escuela tradicional, incluso cuando se refieren al manejo de los símbolos, y operaciones (mentales) cuyo campo de aplicación es más amplio, formando sistemas integrales cuya movilidad -ligada a su reversibilidad- se opone a la estereotipia del desarrollo de los hábitos (El hábito y la operación, pp. 49-60).

Aebli dedica luego una sección a la relación entre la noción de operación y la cooperación de los alumnos (p. 57 sq.). Desarrolla, refiriéndose a Piaget, la cooperación social como uno de los principales agentes formadores en la génesis espontánea del pensamiento infantil, y concluye con la "necesidad imperativa para la enseñanza moderna de sacar partido de ese hecho otorgando un lugar importante a las actividades socializadoras en los programas escolares".

Después del capítulo focalizado en las relaciones entre operaciones y cooperación de los alumnos, el siguiente presenta La investigación, el problema y la construcción de la operación (pp.61-65). Aebli analiza cómo la investigación, por un alumno, de un verdadero problema lleva a la construcción de la operación, y cómo el problema y las preguntas que plantea orientan la investigación y "contienen un esquema anticipador" de la operación intelectual que se realiza (clasificar, ordenar, explicar, etc.). Cuando el esquema anticipador no constituye más que un proyecto global, y que la operación que se va ejecutar, debe diferenciarse y estructurarse durante la investigación, esta conlleva a un progreso del pensamiento, con la construcción de un nuevo esquema sobrepasando la estructura de los esquemas anteriores.

El último capítulo (pp. 66-71) de la Parte psicológica está dedicada a la asimilación en la teoría de Piaget como una "concepción nueva de la aprehensión de la experiencia" para tratar el problema de la relación entre el sujeto asimilador y el objeto asimilado. Aebli se refiere ampliamente a Piaget $(1936,1947)$ y se limita a indicar la evolución de ese proceso que va de los esquemas sensoria-motores "hasta el análisis matemático de los fenómenos y de los objetos".

\section{La aplicación de la didáctica}

Los estudios sobre la investigación, el problema y la construcción de las operaciones mentales fueron retomados en los dos primeros capítulos de la parte didáctica : $L a$ construcción de las operaciones mediante la investigación del alumno (pp. 73-80), y El problema como proyecto de acción (81-85), en el cual Aebli discute el problema como proyecto de acción efectiva, práctica, pero también imaginada. Introduce lo que puede ser el desarrollo de una unidad didáctica con una investigación personal de los alumnos, resaltando la necesidad en ese caso de que el problema sea planteado con "mucho 
cuidado". Se puede pensar aquí en la noción de devolución del problema al alumno, que introducirá años después Guy Brousseau (1986). Aebli también pone de relevancia la importancia de las puestas a punto colectivas, cuyos trabajos en didáctica mostrarán posteriormente que son una dificultad particular para el docente. Este enfoque acerca del estatuto de la investigación de problemas constituye un aporte relevante de Aebli sobre las cuales habrían podrido apoyarse las investigaciones sobre la enseñanza de las matemáticas.

La importancia de la dimensión colectiva introducida en la Parte psicológica tiene su correspondencia en los desarrollos del último capítulo de la Parte didáctica: La cooperación de los alumnos y el "ejercicio operatorio" (pp. 92-106). Aebli distingue en principio dos casos extremos de problemas: aquellos cuya resolución demanda construir una noción u operación nueva -la discusión en común está adaptada- y aquellos que necesitan de la simple aplicación de operaciones conocidas sobre situaciones nuevas, cuya resolución encaja en un trabajo en equipos.

Las siguientes secciones explicitan el trabajo didáctico que aún debe ser efectuado. La elaboración de una nueva noción u operación a partir de la investigación, debe estar seguida por una implementación que debe "[hacer] romper los marcos rígidos de un hábito que podría haberse formado a espaldas del alumno " y “ depurar la operación y hacerla móvil ». Es lo que Aebli denominada “ejercicio operatorio». Las condiciones de la productividad de este ejercicio son luego analizadas : trabajar el lazo entre operación directa y operación inversa (la reversibilidad operatoria), poner en relación operaciones asociativas $\mathrm{y}$, también, poner en relación operaciones y nociones a distinguir : en la didáctica psicológica se trata, para el docente, de hacer trabajar el sistema de operaciones. Finalmente, la interiorización progresiva de las operaciones debe ser organizada vía la representación gráfica de las operaciones. Debe también apuntarse a la implementación de las operaciones interiorizadas por la demanda de repensar la operación ejecutada y luego anticiparla.

En "Didáctica psicológica » se está lejos de la ilusión de un constructivismo estricto que, a veces, se le reprochó a Aebli : el sistema de actividades cognitivas exigidas al alumno, por parte del docente, toma toda su dimensión. Sin embargo, el punto débil del lazo entre psicología y didáctica que propone Aebli es una disminución del proceso de acomodación desarrollado, cuando la aplicación de los esquemas a nuevos objetos conlleva la modificación de los mismos. El autor señala solo en forma breve la existencia y considera que una " psicología didáctica deberá hacer hincapié sobre todo en la relación asimiladora» (p. 67). Ahora bien, numerosos objetos de enseñanza que serán nuevos para el alumno requerirán un cambio conceptual por parte del mismo, con o sin ruptura, que deberá estar organizada por el profesor. La noción subyacente de obstáculo epistemológico ha sido retomada de Bachelard (1938) en el desarrollo de la teoría de situaciones didácticas de Brousseau $(1986,2006)$ y para numerosos didácticos en matemáticas y en ciencias experimentales.

En efecto, la dimensión epistemológica en juego en la didáctica, en tanto tal, no es introducida por Aebli, incluso si una epistemología implícita de las medidas espaciales de perímetro y de superficie, subyace la experiencia que concluye el libro. 


\section{Une experiencia de aplicación de la didáctica psicológica}

La parte experimental que cierra el libro (pp.107-153) presenta una experiencia didáctica dirigida por Aebli en $6^{\circ}$ grado de la enseñanza primaria. Se trata del cálculo del perímetro y de la superficie del rectángulo así como las operaciones inversas. El principio es el de comparar las lecciones organizadas, por el mismo docente, en cursos normales de acuerdo a los principios de la "didáctica tradicional», o según los principios de una "didáctica activa". En ambos casos se plantea el mismo tipo de ejercicios. Se evalúan mediante una comparación los éxitos a un examen inicial, y luego a un examen final (donde los alumnos tienen 100 minutos para resolver 30 problemas, sin dificultades aritméticas). A igual nivel inicial, los alumnos del "grupo moderno" que han beneficiado de una "didáctica activa" tienen más éxito en el examen final. Los alumnos de los subgrupos “inferiores" (8-21 puntos en el examen inicial, más numerosos en el grupo " moderno»), a pesar de sus resultados que son aún bajos, manifiestan menos confusión en las operaciones, lo que se traduce por una mejor distinción de los caracteres, respectivamente, unidimensional del perímetro y bidimensional de la superficie. Los alumnos de los subgrupos "superiores" (22 a 30 puntos en la prueba inicial, más numerosos en el grupo “ tradicional ») tienen la misma calidad (muy elevada) de respuestas en el examen final.

Aebli discute precisamente la interpretación que se puede realizar de la experiencia. Subraya en particular que la evaluación solo captura "el rendimiento intelectual bajo la forma más básica ", en una "prueba incapaz de revelar las influencias más profundas del docente", y que la experiencia de este tipo de enseñanza ha sido demasiado corta. El autor responde también a la posible crítica de la duración de la enseñanza de acuerdo a su didáctica psicológica : la investigación por parte de los alumnos toma más tiempo, en particular en las manipulaciones concretas. Para Aebli, éstas no tienen valor por sí mismas, sino para preparar la representación interiorizada de las operaciones y solo se justifican para los alumnos que él califica como los "menos dotados". El autor responde al dilema que se plantea por la organización del curso mediante una propuesta de " individualización parcial".

\section{La ausencia de relevos y de continuidad de la “Didáctica psicológica » en pedagogía y en didáctica de las matemáticas en Europa francófona : algunas hipótesis}

Las propuestas teóricas y pragmáticas de Aebli no han sido un campo fértil de investigaciones en la enseñanza de las matemáticas; ni siquiera han sido discutidas a fondo, tanto en lo que tenían de productivo, ni en cuanto a sus límites epistemológicos señalados anteriormente.

Se pueden esbozar varias razones, algunas inherentes al contexto francés. A nuestro juicio, obedecen a dos grandes movimientos de los años 50 con relación, por un lado, a la enseñanza de las matemáticas y de la pedagogía, por otro. 
El movimiento de las " matemáticas modernas ", con el " colectivo Bourbaki », generó un debate internacional sobre la renovación de la enseñanza de las matemáticas en la secundaria, implicando a muchos matemáticos de renombre. Gustave Choquet será el primer presidente de la CIEAEM (Comisión internacional para la mejora de la enseñanza matemática), creada formalmente en 1951, resultando significativo que reúna tanto a psicólogos (entre los cuales Piaget y Gonseth), como a matemáticos (como es el caso de Dieudonné, Choquet, Lichnerowicz).

En Francia, estos debates desembocarán en 1968 en la creación de los primeros IREM (Institutos de investigación para la enseñanza de las matemáticas) en el seno de las matemáticas, en la facultad de ciencias de París. Este será el terreno fértil de la didáctica de las matemáticas. Un enfoque como el de Aebli que no se apoya sobre un análisis de los saberes matemáticos, no tenía su lugar.

Del lado de la pedagogía, se desarrolla otro tipo de movimiento epistemológico. En Francia, el jefe de fila es Gastón Mialaret : luego de haber creado en 1946 un laboratorio de psicopedagogía, va a participar en 1953 en una primera reunión, por iniciativa de Robert Dottrens, con investigadores defensores de la cientificidad del dominio, una pedagogía experimental [8]. La siguiente etapa es la separación de las nuevas "Ciencias de la educación " de la psicología, en tres universidades. Datada en 1967, su creación en tanto que disciplina universitaria autónoma en Burdeos, Caen y París es la culminación de las acciones de Gastón Mialaret, Jean Château y Maurice Debesse.

La defensa de Aebli de una didáctica, instrumento de la pedagogía fundada en la teoría psicológica de Piaget, iba a contracorriente de ese movimiento en el cual los actores centrales se situaban en otra línea (tanto a nivel epistemológico como político) : la de Wallon. Para Piaget, el enfoque evolutivo y de construcción de los conocimientos es ante todo cognitivo ; este enfoque es más unitario en Wallon valorizando el rol del Otro y de lo social.

Maurice Debesse ha otorgado a Aebli el crédito de haber abierto la vía de estudios de los efectos de los métodos de enseñanza de las matemáticas, pero es solo la dimensión de la didáctica como método que es puesta de relevancia (1955, pp. 802-803). Antoine Léon, convocado en 1957 por Debesse a la Sorbonne [ ${ }^{9}$, discutiendo luego acerca de " la psicología como fundamento de toda acción educativa ", sintetiza esta primera obra de Aebli diciendo que " se inspira de las concepciones de J. Piaget para implementar una pedagogía donde la formación de las nociones es considerada como una construcción psicológica, apelando a la actividad de investigación y alcanzando las operaciones móviles, integradas en sistemas de conjunto" (Léon, 1966, p. 464) - pero cita luego a Vinh Bang et Morf, colaboradores próximos de Piaget- a fin de limitar el alcance de la influencia que puede ejercer la psicología en el desarrollo de la pedagogía.

En cambio, al abogar "Por una educación científica" en el primer número de la recién aparecida Revue française de Pédagogie (1967), Jean Château lamenta que "la psicología de la educación se encuentre en un estado embrionario ", sin realizar ninguna mención de Aebli. Durante el mismo período, no se registrará ninguna referencia a Aebli en el libro editado por Mialaret "L'apprentissage des mathématiques", sin embargo subtitulado "Essai de psycho-pédagogie".

A los matemáticos que reivindican la iniciativa epistemológica en la enseñanza de su disciplina, a los pedagogos que afirman su autonomía respecto de la psicología, se les une también un psicólogo, Pierre Gréco, colaborador estrecho de Piaget, -quien también retoma sus cursos en la Sorbona- e interlocutor de Lichnérovicz [10] : " La idea 
de una pedagogía que sería una psicología aplicada a la educación, me parece un claro ejemplo de una aberración epistemológica [...] Sobre la naturaleza del saber matemático a trasmitir, es primero el matemático quien tiene la palabra."

\section{La coyuntura en Francia no dejaba lugar definitivamente para una "Didáctica psicológica »}

Con la constitución de la didáctica de las matemáticas en Francia en los diez años siguientes, las relaciones con la psicología serán redefinidas. Maestro de escuela primaria y luego matemático, Guy Brousseau presenta un panorama bien claro de la historia de su teoría con su origen matemático y sus fuentes psicológicas en un artículo reciente : "De los dispositivos piagetianos... a las situaciones didácticas " (2012). Psicólogo, y también habiendo realizado su tesis con Piaget, Gérard Vergnaud es junto a él uno de los actores de la creación de la comunidad de didáctica de las matemáticas. Su teoría de los campos conceptuales (1990) da en los aprendizajes todo su lugar tanto a los objetos matemáticos como al desarrollo del alumno -luego acentuará el rol central de los esquemas. Se trata del desarrollo de otra historia, que verá venir en el cambio de siglo una nueva articulación de la didáctica de las matemáticas con la psicología [11], dando lugar tanto a la actividad del alumno como a la del docente. Pero Aebli y su "Didáctica psicológica» han sido - injustamente - olvidados en el debate epistemológico sobre la didáctica.

Bibliografia

Aebli, H. (1951). Didactique psychologique. Application à la didactique de la psychologie de Jean Piaget. (4ème édition 1966). Neuchâtel, CH : Delachaux \& Niestlé.

Aebli, H. (1953). Dittatica psicologica. Applicazione alla didattica della psicologia di Jean Piaget.P Firenze : Giunti Barbera.

Aebli, H. (1958). Una didáctica fundada en la psicología de Jean Piaget. Buenos Aires: Kapeluz.

Aebli, H. (1963). Psychologische Didaktik. Stuttgart, Germany : Klett.

Aebli, H. (1965). Psychologickádidaktika. Hradec Kr.lov. : PdF KU.

Aebli, H. (1966/1971). Didática psicológica : aplicação à didática da psicologia de Jean Piaget. São Paulo : Companhia editora nacional.

Aebli, H. (1973).Didactica psihologică. Bucuresti : EdituraDidactică.

Aebli, H. (1982). Dydaktyka psychologiczna. Zastosowanie psychologii Piageta do dydaktyki. Warszawa : PWN.

Aebli, H. (1960). Über die geistige Entwicklung des Kindes. Habilitation. Université de Zurich. (publication 1963).

Aebli, H. (1980). A evolução mental da criança. Petrópolis : Vozes.

Aebli, H. (1961). Grundformen des Lehrens. Ein Beitrag zur psychologischen Grundlegung der Unterrichtsmethode. Stuttgart: Klett.

Aebli, H. (1965). I principi fondamentali dellinsegnamento. (Trard. Mario Chiarenza). Firenze : Universitaria Barbera. 
Aebli, H. (1976) Grundformen des Lehrens. Eine Allgemeine Didaktik auf kognitionspsychologischer Grundlage (éditionlargementrevue et élargie du texte de 1961). Stuttgart : Klett.

Aebli, H. (1978). Von Piagets Entwicklungspsychologie zur Theorie der kognitiven Sozialisation. In Steiner, G. (Hrsg.), Die Psychologie des 20. Jahrhunderts. Band VII : Piaget und die Folgen (pp. 604-627). Zürich : Kindler.

Aebli, H. (1980). Denken: Das Ordnen des Tuns. Band 1, Kognitive Aspekte der Handlungstheorie. Stuttgart: Klett-Cotta.

Aebli, H. (1981). Denken: Das Ordnen des Tuns. Band 2, Denkprozesse. Stuttgart: Klett-Cotta.

Aebli, H. (1983). Zwölf Grundformen des Lehrens. Eine Allgemeine Didaktik auf psychologischer Grundlage. Stuttgart: Klett-Cotta.

Aebli, H. (1987). Grundlagen des Lehrens (vol. 2). Stuttgart: Klett-Cotta.

Aebli, H. (1988). 12 formas basicas de enseña. Madrid : Narcea.

Aebli, H. (1991). Factores de la enseñanza que favorecen el aprendizaje autonomo(Trad. R. Lucio). Madrid : Narcea.

Bachelard, G. (1938). La formation de l'esprit scientifique. Paris : Vrin.

Brousseau, G. (1986). Théorisation des phénomènes d'enseignement des mathématiques. Thèse de Doctorat d'État es Sciences. Université de Bordeaux.

Brousseau, G. (2012). Des dispositifs piagétiens ... aux situations didactiques. Éducation et Didactique, 6(2), 103-129.

Château, J. (1967). Pour une éducation scientifique. Revue Française de Pédagogie, 1, 9-16. Debesse, M. (1955). Les méthodes pédagogiques. In H. Piéron (Éd.), Traité de psychologie appliquée (t.4 : Formation humaine). Paris : PUF.

Léon, A. (1966). Psychologie et action éducative : la notion de psychopédagogie. L'Année Psychologique, 66(2), 461-474.

Mialaret, G. (1967). L'apprentissage des mathématiques. Essai de psycho-pédagogie.

Bruxelles : Charles Dessart.

Ouvrier-Bonnaz, R. \& Lacomblez, M. (2014). Entre schème et didactique(s) : une mise en perspective historique pour penser la question des référentiels d'activité professionnelle. In B. Prot (Éd.), Les référentiels contre l'activité. Toulouse : Octarès. Piaget, J. (1936). La naissance de l'intelligence chez l'enfant. Neuchâtel : Delachaux et Niestlé.

Piaget, J. (1975). L'équilibration des structures cognitives. Problème central du développement. (Études d'Épistémologie Génétique XXXIII). Paris : PUF.

Vergnaud, G. (1990). La théorie des champs conceptuels. Recherches en Didactique des Mathématiques, 10(1), 133-170.

\section{NOTAS}

1. [1] Como lo muestra la bibliografía de Aebli, su primera obra « Didáctica psicológica..." fue ampliamente traducida en Europa y América Latina. Sus obras posteriores sobre el desarrollo del 
pensamiento del niño, la educación y la formación de los maestros, fueron publicadas en alemán y jamás fueron traducidas al francés, y al parecer tampoco al inglés.

2. En tanto que excepción, Michel Fabre desarrolló la noción de "problematización" como vía de educación en particular en el campo de las ciencias en referencia a Aebli, cuya didáctica psicológica (obra desconocida, poco citada y que sin embargo elaboró los principales conceptos de una teoría de las situaciones-problemas) podría servir de puente entre una psicología de la actividad y una epistemología del problema." Cf. Fabre, M., \& Musquer, A. (2009). Comment aider l'élève à problématiser ? Les inducteurs de problématisation. Les Sciences de l'Éducation, 42(3), 111-129.

3. Catedrático sucesivamente de las universidades de Zürich, $\mathrm{CH}$;Saarbrücken, RFA; Berlin, RFA; Constanze, RFA; Berne, $\mathrm{CH}$

4. La única referencia explícita de Brousseau $(1986,2006)$ a " Didactique psychologique..." será la denuncia de la concepción sensualista-empirista mediante la cual se le une a Aebli.

5. Lay, W.A. (1914, 3era ed.). Der Rechunterricht auf experimentell-pädagogischerGrundlage. Leipzig.

6. Aebli también hace referencia a Claparède cuya teorización es menos elaborada que la de Dewey. Analiza brevemente la "Didactique discipline mentale" de Kerschensteiner y critica su distinción radical entre funciones prácticas y funciones teóricas del saber, que revelan un profundo dualismo entre acción y pensamiento.

7. Nota de la traductora: Las paginas corresponden a la versión francesa, sin embargo existe una traducción en español: Hans Aebli, Una didáctica fundada en la psicología de Jean Piaget. Buenos Aires, Ed. Kapelusz, 1973.

8. Mialaret presidiría en 1958 la AIPELF (Asociación internacional de pedagogía experimental de lengua francesa), creada nuevamente. En cuanto a Dottren, por ese entonces será el director del Laboratorio de pedagogía experimental de la Universidad de Ginebra fundado por él y del cual, Aebli formaba parte.

9. Luego de su exclusión del INETOP por divergencias con Piéron sobre la psicopedagogía de la orientación profesional.

10. Gréco, P. (1969). Note sur la coopération des psychologues et des enseignants dans le cadre des expériences didactiques. Bulletin de l'APMEP, 206, 27-32.

11. El enfoque instrumental de Rabardel alimenta el estudio de las tecnologías en la enseñanza de las matemáticas (Artigue, Lagrange). El doble enfoque de didáctica y psicología evolutiva de Robert \& Rogalski se encuentra en el centro de estudios de las prácticas de docente.

\section{AUTOR}

\section{JANINE ROGALSKI}

Directeur de recherche CNRS honoraire, associée au Groupe de Recherche et d'Etude sur l'Histoire du Travail et de L'Orientation (GRESHTO), Centre de Recherche sur le Travail et le Développement (CRTD), Conservatoire National des Arts et Métiers (CNAM), 41, Rue Gay Lussac 75005, Paris, France

Janine.Rogalski@univ-paris8.fr 